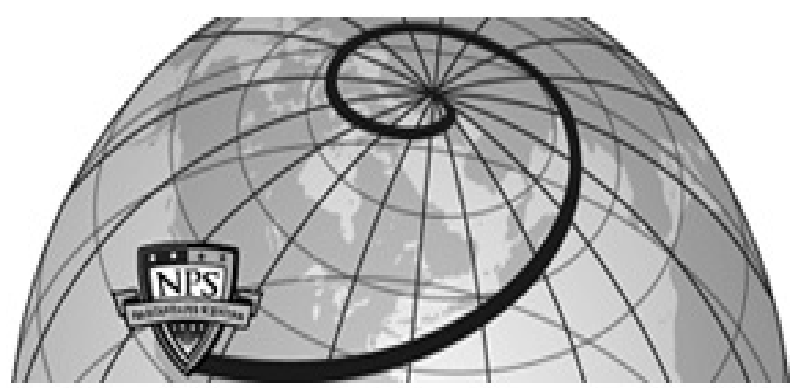

Calhoun: The NPS Institutional Archive DSpace Repository

\title{
Analysis of Alternative Rework Strategies for Printed Wiring Assembly Manufacturing Systems
}

Driels, Morris; Klegka, John S.

IEEE Transactions on Components, Hybrids, and Manufacturing Technology, Vol. 14, No. 3, September 1991.

https://hdl.handle.net/10945/42554

This publication is a work of the U.S. Government as defined in Title 17, United States Code, Section 101. Copyright protection is not available for this work in the United States.

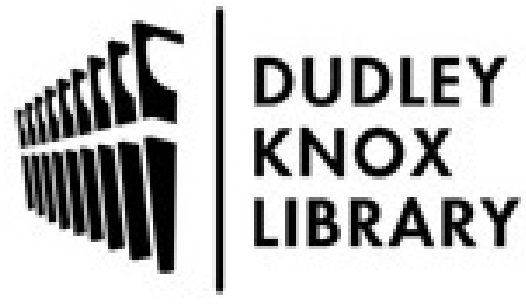

http://www.nps.edu/library
Calhoun is the Naval Postgraduate School's public access digital repository for research materials and institutional publications created by the NPS community. Calhoun is named for Professor of Mathematics Guy K. Calhoun, NPS's first appointed -- and published -- scholarly author.

Dudley Knox Library / Naval Postgraduate School 411 Dyer Road / 1 University Circle Monterey, California USA 93943 


\title{
Analysis of Alternative Rework Strategies for Printed Wiring Assembly Manufacturing Systems
}

\author{
Morris Driels, Member, IEEE, and Major John S. Klegka
}

\begin{abstract}
This paper presents a model for predicting the cost of test, diagnosis, and rework activities in the manufacture of printed wiring assemblies (PWA's). Rework is defined as all actions taken to correct or improve the basic assembly process. These actions may include those of inspectors and solder touchup technicians who do not add value to the PWA, but whose actions are required in order to produce acceptable yields from the manufacturing process. Two alternative rework strategies for contemporary PWA manufacturing systems are presented: terminal rework and distributed rework. Rework may occur after all assembly operations have been accomplished (terminal rework) or it may be distributed throughout the assembly process. This paper analyzes the economic basis for deciding between the two rework strategies. The paper assumes that the only reason for utilizing distributed rework is to reduce the cost of producing acceptable PWA's, otherwise the cost of the distributed rework effort cannot be justified. The paper presents a model of each rework strategy. The effect of each strategy upon first pass yield (FPY) of the manufacturing process is discussed. The effect on FPY is then used to evaluate the economic benefit of each rework strategy as an aid in deciding which strategy to use. The increase in FPY needed to justify distributed rework is calculated.
\end{abstract}

\section{PWA Manufacturing Systems}

A WIDE variety of printed wiring assembly (PWA) manufacturing systems may be observed throughout the industry. These systems have been developed to operate efficiently in specific areas of the PWA manufacturing environment as determined by factors such as technology, production volume, design complexity, component types, and product life span. In the area of surface mount technology (SMT), for example, considerable standardization has been achieved in interconnect methods, component shapes and packaging, and substrate design. As a result, a wide variety of SMT products are manufactured using very similar assembly systems. In order to accurately predict the costs associated with operating these systems, they must be modeled on a detailed basis. To obtain the needed detail, models have been developed in which the total system is decomposed into individual modules. The individual modules are analyzed to model the cost and performance of each station [1], [2].

Manuscript received May 14, 1990; revised April 10, 1991

M. Driels is with the Department of Mechanical Engineering, Naval Postgraduate School, Monterey, CA 93943.

Major J. S. Klegka is with the Department of Civil and Mechanical Engineering, United States Military Academy, West Point, NY 10996.

IEEE Log Number 9101464.
A PWA manufacturing system usually consists of a series of automated and manual assembly and process stations. The stations may be connected by an automatic or manual transfer device which moves the partially completed PWA's through the system. Each PWA follows a relatively fixed path through the manufacturing system. A typical assembly system consists of a solder paste dispenser, one or more automatic component placement machines, one or more manual inspection and placement stations, a solder reflow station, a cleaning station, and a test, diagnosis, and rework station [8], [9], [11]

\section{Manufacturing Cost Model}

The manufacturing cost model has the form shown below [4]. $C_{\mathrm{pr}}$, the cost of manufacturing one PWA, is given by

$$
C_{\mathrm{pr}}=C I+C A+C T D R
$$

where

$C I$ sum of all inventory terms,

$C A$ sum of all assembly terms,

$C T D R$ sum of all test, diagnosis, and rework terms.

These cost terms are analyzed in detail in [1], [2]. The general expression for the assembly cost model is described. We will look at this model briefly here, as it is the basis of both the assembly cost terms and the test, diagnosis, and rework cost terms.

\section{Assembly Cost Terms ( $C A$ )}

The total assembly cost per completed assembly, $C A$, is composed of terms to account for the cost of setup and assembly at each station of a multistation assembly system. Thus

$$
\mathrm{CA}=\sum_{i=1}^{s} \mathrm{CA}_{i}
$$

where $s$ is the number of stations in the assembly system and $\mathrm{CA}_{i}$ is the setup and assembly cost incurred at assembly station $i$.

To develop a cost model for a PWA assembly system, we must develop cost terms for each station. The general form for these cost terms is

$$
\mathrm{CA}_{i}=t_{\mathrm{pr}_{i}}\left(W_{t_{i}}+\frac{M_{i}}{7200000 N S}\right)
$$


where

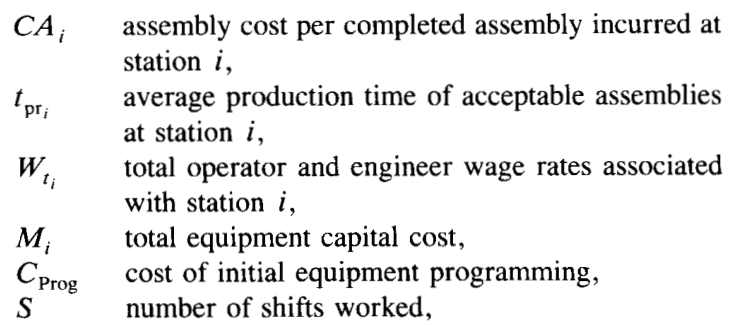

$N \quad$ number of years in the payback period. Note that 50 weeks per year times $40 \mathrm{~h}$ per week times $3600 \mathrm{~s} / \mathrm{h}$ yields $7200000 \mathrm{~s}$ per shift-year.

Although this model follows the concepts developed by Boothroyd [5], the general form of the assembly cost equation, shown above, must be adapted to each station considered [1], [2].

Assembly cost at each station is the product of the time to assemble, $t_{\mathrm{pr}}$, and the wage rate of the personnel and equipment involved at the station, $\left(W_{t}+\mathrm{M} / 7200000 \mathrm{NS}\right)$. While the costs of some support personnel (an engineer or a control programmer, for example) can be included if they are involved with the station on a regular basis, in general, overhead costs are not included in this model. These indirect costs must be included at each station by the analyst using whatever method is appropriate for the firm. For a discussion, see [7].

\section{Test, Diagnosis, and Rework Cost Terms} (CTDR)

Once all assembly operations are complete, PWA's move to the test facility where some of the PWA's will receive a test cycle. This cycle may consist of an in-circuit test, a functional test, or a combination. The proportion tested depends on the cost, complexity, and intended use of the PWA. In general, as the boards become more expensive and more complex, the proportion being tested will increase. For example, in the computer and communications industries, usually all PWA's are tested.

In this model we assume that all PWA's undergo an in-circuit test cycle. Satisfactory boards are installed in systems while faulty boards must be diagnosed and reworked. This cycle is illustrated in Fig. 1. The goal is to fix all detected faults. Detected faults include the following.

1) An incorrect SMC that is successfully assembled.

2) An improperly assembled SMC, one in which the electrical connection has not been made during the soldering process.

3) A faulty SMC that fails immediately or is so far out of tolerance that it behaves as an incorrect SMC.

Since each PWA is tested there is a common test cost which we assume to be constant for each PWA of a given design produced on the same line. This cost, $C_{\text {Test }}$, depends on the time the test takes and on the cost of the test

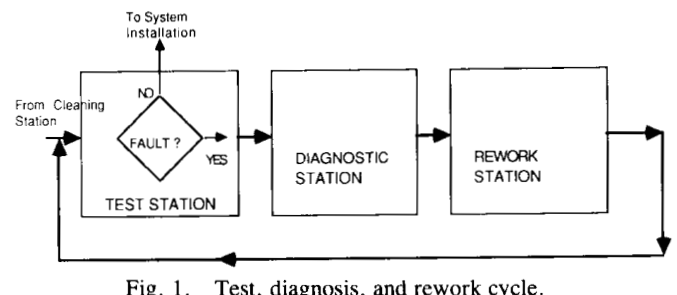

Fig. 1. Test, diagnosis, and rework cycle.

equipment. Using our model we have

$$
C_{\text {Test }}=t_{\text {Test }}\left\{W_{\text {Test }}+\frac{\left(M_{\text {Test }}+C_{\text {prog }}\right)}{(7200000 N S)}\right\}
$$

where

$M_{\text {Test }}$ total cost of test equipment (computer, fittings, transfer equipment, etc.),

$W_{\text {Test }}$ wage paid to test operator (in dollars per second),

$t_{\text {Test }}$ average time to conduct test (in seconds),

$C_{\text {Prog }}$ cost of initial test software development,

$N$ number of years in the payback period,

$S \quad$ number of shifts worked.

The diagnosis and rework costs are determined by the fault rate for all components, the number of components in the PWA, and the cost of operating the diagnostic and rework stations. In addition, after the rework is completed, another test is conducted to verify that the initial faults have been corrected and that no new faults have been introduced. We will assume that virtually all faults are corrected successfully during the rework step.

For a given PWA design, the probability of developing a fault during the manufacturing process increases with the number of components on each PWA. We define the first pass yield (FPY) to be the fraction of PWA's which pass the first circuit test. FPY is given by

$$
\mathrm{FPY}=(1-F R)^{n}
$$

where 1) $F R$ is the fault rate, the proportion of components that cause a fault. The fault rate may be obtained from historical data for an existing process or it may be estimated for a new process or product design. The fault rate will have a number of elements including component placement accuracy, component reliability, and the expected solder process yield and the number of component leads. 2) $n$ is the number of components on each PWA.

Consequently, the proportion of PWA's that fail the first circuit test is 1 - FPY. All these PWA's must be diagnosed and reworked.

If the average cost to diagnose and rework a faulty PWA is known, the rework cost per PWA may be calculated. Since each reworked PWA must be tested again, the diagnosis and rework cost, $C D R$, is given by

$$
C D R=\left(C P F+C_{\text {Test }}\right)(1-\text { FPY }) .
$$

where

$C D R$ diagnosis and rework cost per rejected PWA with $n$ components, 
$C P F$ average cost of diagnosing and reworking one failed PWA (in dollars). A typical value is $\$ 50$ [4],

$C_{\text {Test }}$ cost of running the test on the reworked PWA (assumed to be the same as the original test).

Thus the test, diagnosis, and rework costs are

$$
C T D R=C_{\text {Test }}+C D R \text {. }
$$

At this point it is useful to compare the relative magnitude of the various manufacturing cost elements. In the next section a simulation of the typical manufacturing system is conducted.

\section{AsSEmbly System ANALysis}

The preceding equations, defining the performance and costs involved in PWA assembly, have been modeled using a spreadsheet technique. A detailed description of the system modeled and the equations used are shown in [1], [2]. The model takes the form of a Lotus 1-2-3 template, allowing changes in system parameters to be easily made and the effect on manufacturing costs to be readily observed. This spreadsheet software was chosen due to its widespread availability in the business world as well as its ease of use.

Using estimated product data obtained from Boothroyd [5], [6], Russell [3], [4], and industrial companies using SMT assembly systems, some interesting trends may be observed. For example, the model indicates that for a typical assembly (lot size $=20$ ) the major cost terms are:

1) inventory cost $=\$ 0.27$;

2) assembly cost $=\$ 7.82$;

3) test and rework cost $=\$ 21.88$.

This confirms the results obtained by Russell [4], namely, that the test, diagnosis, and rework cost is a significant part of PWA manufacturing cost. Typical data used in this simulation can be found in [1], [2]. This situation is graphically illustrated in Fig. 2.

Based on this simulation, test, diagnostic, and rework costs seem to be significant elements of the total manufacturing cost. Depending on lot size, $C T D R$ may account for a half to three quarters of the manufacturing cost per PWA. Due to the apparent importance of this term, it is appropriate to examine it in more detail as a source of potentially major savings.

\section{Detalled Diagnostic and Rework Costs}

Based on the results discussed in the previous section, it is evident that test, diagnosis, and rework costs can be significant elements of total PWA manufacturing costs. These costs are undesirable since test and rework activities do not add value to the PWA being produced. The activities are required to avoid sending faulty products to the customer. The first reaction to this issue is to look for ways to improve either the manufacturing process or the product design to eliminate the need for test and rework. However, the current and foreseeable state-of-the-art in this industry seems able to produce solder joints with a defect rate in the range of 100 parts per million (ppm) [8]. Here defective joints are defined to be

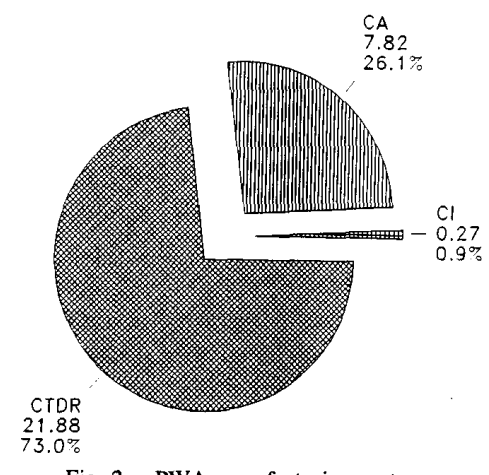

Fig. 2. PWA manufacturing costs.

those which cause electrical failure, or customer rejection due to cosmetic reasons. Unfortunately, this defect rate implies that, for a PWA with 2000 solder joints, almost $20 \%$ of the assemblies produced will have at least one defective solder joint. Other defects, such as faulty components, only add to the problem. Thus test, diagnosis, and rework must be considered as part of the manufacturing process. This area is one where potentially large savings could be realized.

A model of test cost was presented previously. This section examines the diagnosis and rework issue in more detail. Current practices followed in the PWA manufacturing industry are discussed. The diagnosis and rework process is broken into its component parts and each is considered separately. A cost model is developed for each step of the process.

\section{A. Diagnostic Costs}

Due to the difficulty in testing PWA's which contain surface mounted components, there may be a separate diagnostic station. The cost of operating the diagnostic station, $C_{\text {Diag }}$, is just

$$
C_{\text {Diag }}=t_{\text {Diag }}\left\{W_{\text {Diag }}+\frac{\left(M_{\text {Diag }}+C_{D . P r o g}\right)}{(7200000 N S)}\right\}
$$

where

$M_{\text {Diag }}$ total cost of diagnostic equipment (computer, fittings, transfer equipment, etc.),

$W_{\text {Diag }}$ wage paid to diagnostic technician,

$t_{\text {Diag }}$ average time to diagnose a faulty PWA,

$C_{D \text {.Prog }}$ cost of initial diagnostic software development.

The goal of the diagnostic station is to provide precise information to the rework technician/station where the actual repair is made.

\section{B. Rework Costs}

In contrast with the wide variety of assembly processes used in PWA manufacture, current rework methods are almost entirely manual and are quite similar. A highly skilled technician is required to position the faulty component under a reflow device which heats the solder joints until the solder is molten. The faulty component is then either repositioned or 
replaced. If the component is to be removed and replaced, some solder site preparation must occur at the circuit board pads. This preparation includes cleaning the solder pads and placing new solder paste. After reflowing the solder, the technician then inspects and cleans the PWA and sends it to the test station to make sure the fault has been corrected and no new fault has been introduced [8], [12], [13]. Fig. 3 shows a schematic representation of this process. This whole operation can be quite time consuming and is subject to wide variability. The technician is typically provided with a number of devices to assist him in making the appropriate repairs without introducing faults in adjacent components. Thus the cost to rework one faulty PWA, $C_{\mathrm{RW}}$, becomes

$$
C_{\mathrm{RW}}=t_{\mathrm{RW}}\left\{W_{\mathrm{RW}}+\frac{M_{\mathrm{RW}}+C_{R . \text { Prog }}}{(7200000 N S)}\right\}
$$

where

$$
\begin{array}{ll}
M_{\mathrm{RW}} & \begin{array}{l}
\text { total cost of rework equipment (reflow device, } \\
\text { controller, fittings, inspection equipment, part } \\
\text { feeders, transfer equipment, etc.), }
\end{array} \\
W_{\mathrm{RW}} & \text { wage paid to rework technician(s), } \\
t_{\mathrm{RW}} & \text { average time to rework a faulty PWA, } \\
C_{R . P r o g} & \text { cost of initial reflow controller programming. }
\end{array}
$$

Although the rework technician has devices to assist him in performing the rework task, the rework process can introduce a new fault in a number of ways. The technician may damage the component he is working on or an adjacent component. This damage may or may not be observable to the technician. Those faults which the technician detects at the rework station will be repaired at the station. Those faults which are not detected will be identified when the reworked board goes back to the in-circuit tester to be tested again. In addition, the technician may work on the wrong component, failing to fix the fault. To include the effect of these errors on the rework cost, we express the average rework time as

$$
t_{\mathrm{RW}}=t_{\mathrm{SU}}+t_{R}+x_{A} T_{A}+x_{B} T_{B}
$$

where

$t_{\mathrm{SU}} \quad$ setup time,

$t_{R}$ repair time (preheat, remove old component, attach new component, inspect PWA, etc.),

$x_{A}$ proportion of replacement components that the technician can identify as faulty (damaged, incorrect, etc.) prior to rework,

$T_{A}$ time for technician to get new part,

$x_{B}$ proportion of reworked PWA's wherein the technician introduces a fault which is detected at the rework station,

$T_{B}$ time to fix fault at rework station (approximately $t_{R}$ ).

The effect of a faulty PWA leaving the rework station is considered below.

Now, since each reworked PWA must be tested again, the diagnostic and rework cost, $C D R$, is given by

$$
C D R=\left(C_{\text {Diag }}+C_{\mathrm{RW}}+C_{\text {Test }}\right)(1-\mathrm{FPY})
$$

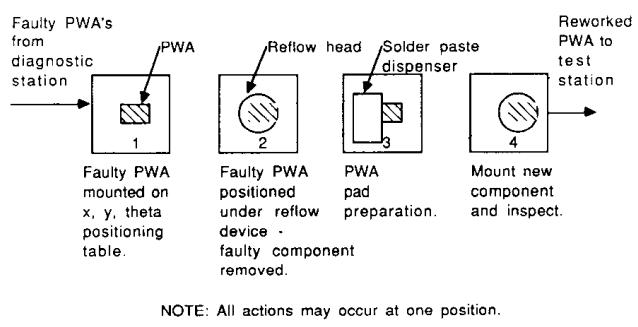

Fig. 3. Contemporary rework process.

where

$C D R$ diagnostic and rework costs per PWA with $n$ components,

$C_{\text {Diag }}$ average cost of diagnosing one failed PWA (in dollars),

$C_{\mathrm{RW}}$ average cost of repairing one failed PWA (in dollars),

$C_{\text {Test }}$ cost of running the rest on the reworked PWA (assumed to be the same as the original test)

At this point the effectiveness of the rework process must be considered. We define $x_{C}$ to be the proportion of reworked PWA's, wherein the technician either introduces a fault which is not detected at the rework station or else fails to correct the specified fault. An example of the latter error would occur when the technician replaces a good component by mistake, leaving the faulty component on the board. This term may also account for incorrect fault information being provided to the rework technician. If $x_{C}$ is known it may be used directly, otherwise, it may be estimated as shown below. Let $P_{\mathrm{I}}$ be the proportion of PWA's wherein the fault(s) have been correctly identified by the diagnostic station; $P_{\mathrm{II}}$ be the proportion of PWA's wherein the technician works on the designated fault(s) and no others; and $P_{\text {III }}$ be the proportion of PWA's wherein the technician performs a successful rework operation. Then no new faults are introduced. Then the probability that a reworked PWA is free of faults is $P_{\mathrm{I}} \times P_{\mathrm{II}} \times P_{\mathrm{III}}$ (see Fig. 4) and the probability that it contains a fault, $x_{C}$, is

$$
x_{C}=1-\left(P_{\mathrm{I}} \times P_{\mathrm{II}} \times P_{\mathrm{III}}\right)
$$

where $x_{C}$ is the proportion of reworked PWA's which contain a fault, from whatever cause, when they return to the test station.

The fault will be detected when the PWA is retested. At this point the PWA must begin again the diagnostic-reworkretest cycle. The cost of this second cycle should be the same, on average, as the first cycle. The proportion of PWA's that receive this second cycle is $(1-$ FPY $)\left(x_{C}\right)$. Thus the cost of this second rework effort again spread out over all PWA's manufactured is

$$
C D R_{2}=\left(C_{\text {Diag }}+C_{\mathrm{RW}}+C_{\text {Test }}\right)(1-\mathrm{FPY})\left(x_{C}\right) \text {. }
$$

Similarly, if a third rework cycle is required, the proportion of PWA's which undergo this cycle is $(1-\mathrm{FPY})\left(x_{C}\right)^{2}$ and the cost penalty per acceptable assembly is

$$
C D R_{3}=\left(C_{\text {Diag }}+C_{\mathrm{RW}}+C_{\text {Test }}\right)(1-\mathrm{FPY})\left(x_{C}\right)^{2} \text {. }
$$




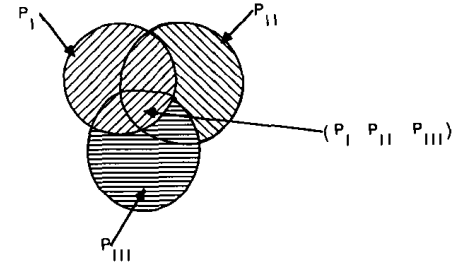

Fig. 4. Probability of a fault-free PWA.

Clearly these incremental cost effects are significant for large values of $x_{C}$, i.e., $x_{C}>0.1$. For $x_{C}<0.01$, the subsequent rework cycles have negligible effect. For $0.01<$ $\bar{x}_{C}<0.1$, the effect of subsequent rework may or may not be significant.

Continuing to write out terms for $C D R_{n}$ one obtains a geometric series, the general $(i$ th) term having the form

$$
C D R_{i}=\left(C_{\text {Diag }}+C_{\mathrm{RW}}+C_{\text {Test }}\right)(1-\mathrm{FPY})\left(x_{C}\right)^{i-1} \text {. }
$$

For $n$ terms, the series has the form [14]

$$
\sum_{i=1}^{n} C^{\prime}\left(x_{C}\right)^{i-1}=\frac{C^{\prime}\left(x_{C}^{n}-1\right)}{x_{c}-1}
$$

for $x_{C} \neq 1$ where $C^{\prime}=\left(C_{\text {Diag }}+C_{\mathrm{RW}}+C_{\text {Test }}\right)(1-$ FPY $)$.

For $x_{C}=1$, one has the case where each rework operation introduces new faults into the reworked PWA. Clearly, in this case, the rework process is incapable of performing the rework function, and rework is not an option. In this event, faulty PWA's must be scrapped and their cost borne by the acceptable PWA's produced. This situation is beyond the scope of this study and will not be considered further.

For $x_{C}>1$ one would have the case where each rework operation introduced more faulty PWA's into the rework process. This case violates physical constraints, since the rework station can only affect PWA's that go through the rework process. It cannot generate new, faulty boards. Thus for a rework system to exist, $x_{C}$ must lie in the range $0 \leq x_{C}<1$ where $x_{C}=0$ indicates a perfect rework process and as $x_{C}$ approaches 1 the rework process becomes less and less effective.

For $0 \leq x_{C}<1$ the geometric series in (16) converges [14]. For $n=\infty$ :

$$
\sum_{i=1}^{\infty} C^{\prime}\left(x_{C}\right)^{i-1}=\frac{C^{\prime}}{1-x_{C}} .
$$

This expression gives the cost per acceptable PWA of allowing a faulty PWA to be reworked as often as needed. As such, it provides an upper limit on the cost penalty associated with rework.

In practice, however, a PWA will be reworked only a finite number of times. There are several reasons for this.

1) Every rework cycle degrades the long term reliability of the PWA. Repeatedly reworked boards will fail sooner under field conditions.

2) A PWA will only be reworked up to some fraction of its economic value. There is a rework budget for each PWA.
3) There may be some subtle fault in the PWA. When one fault is fixed, another appears. This situation is found with some logic boards. In this case, the PWA could get reworked forever.

A typical value for $n$ is $n=3$.

$C T D R$ is the cost of operating the test, diagnosis, and rework stations spread out over all PWA's manufactured by the system. Here we assume that virtually all reworked PWA's pass the second circuit test. Thus there is no need for additional rework and retest activities. If this assumption is invalid, then (11) must be modified to include the cost of those additional activities.

For each acceptable PWA, the test, diagnosis, and rework cost, $C T D R$, becomes

$$
C T D R=C_{\text {Test }}+C D R .
$$

The detailed cost model for $C T D R$ was implemented on a Lotus 1-2-3 spreadsheet to attempt to validate the values for cost per fault (CPF) used previously. In this model it is assumed that it takes $14 \mathrm{~min}$ to diagnose the fault and $15 \mathrm{~min}$ to rework the fault. Based on the values assumed [1], [2], the detailed model yielded a value of $\$ 41.95$. This value is very close to the $\$ 50$ assumed earlier. Thus the model seems to perform as intended by identifying the source of the direct cost elements. When one compares the value of CTDR to $\mathrm{CA}$, one again notes that the test, diagnosis, and rework steps may contribute as much as a half of the total manufacturing cost.

The next section examines the effect of having the rework effort located at different points in the manufacturing process. A model is developed to predict the costs and benefits associated with various configurations.

\section{Terminal versus Distributed Rework}

The goal of any manufacturing process is to produce a high yield of acceptable assemblies. As noted previously, the limits of the PWA manufacturing process may well result in a significant proportion of PWA's which have a defect of some sort. Unless the defective PWA's are to be discarded, they must be reworked before being sent to the customer.

Two approaches to the rework issue are apparent in industrial practice: terminal rework and distributed rework. In terminal rework, all rework activities are concentrated at the end of the manufacturing line. PWA's proceed through the manufacturing process and are tested. Faulty boards may go to a diagnostic station and are then reworked. Terminal rework is illustrated in Fig. 5. In a distributed rework environment, on the other hand, some degree of rework may occur after each assembly station, with final rework located after the test station. There are many possible configurations for a distributed rework system. One possible configuration is illustrated in Fig. 6. Note that the distributed rework system includes a terminal rework station. This terminal rework station probably has the same capabilities in both environments.

Each strategy has advantages and disadvantages. The terminal rework approach concentrates all rework activities at a
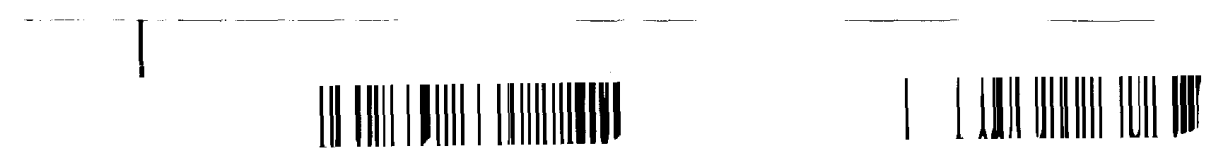


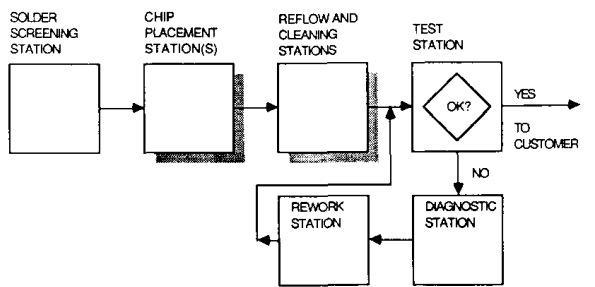

Fig. 5. Terminal rework process.

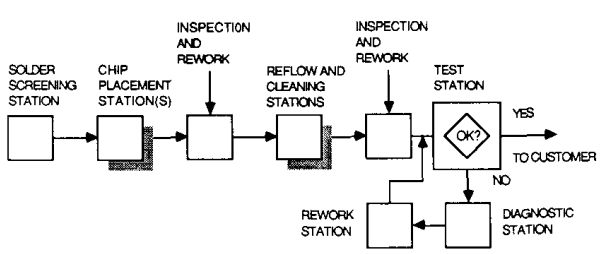

Fig. 6. Distributed rework process.

single location and may allow easier control of the rework process. Only faulty PWA's go through the rework process. However, this approach ensures that all rework activities will involve reflowing some solder joints, since the PWA has already been through the solder station.

The distributed rework approach allows minor, easily performed rework actions to take place throughout the manufacturing process. Rework occurs closer to the point where the fault occurred. For example, an out of position component may be readily pushed into place before encountering the solder station. Faulty assembly machines can be identified more quickly, preventing the production of large numbers of faulty PWA's which must then be reworked. On the other hand, the use of a distributed system may require more technicians. Unless the number of technicians is adequate, the distributed rework stations could form bottlenecks which would reduce the production capacity of the assembly system.

In deciding which strategy to follow, it is necessary to know the costs associated with each. Thus the cost of rework must be assessed on a per assembly basis.

\section{A. Terminal Rework}

The cost model developed in Section VI describes a situation in which all rework activities are concentrated in one station. This situation describes a terminal rework environment. The cost model for this rework strategy is shown below:

$$
C_{\mathrm{RW}_{T}}=t_{\mathrm{RW}}\left\{W_{\mathrm{RW}}+\frac{M_{\mathrm{RW}}+C_{R \cdot \mathrm{Prog}}}{(7200000 N S)}\right\} .
$$

Recall that $C_{\mathrm{RW}}$ is the cost to rework one faulty PWA.

The total rework process includes the diagnostic, rework, and retest activities performed on faulty PWA's. Since only faulty PWA's undergo this rework process, the cost penalty on a per PWA basis for diagnosis, rework, and retest is

$$
C D R_{T}=\left(C_{\text {Diag }}+C_{\mathrm{RW}_{T}}+C_{\text {Test }}\right)\left(1-\mathrm{FPY}_{T}\right) \text {. }
$$

The subscript $T$ denotes terminal rework. Note that $x_{C}<$ 0.01 is assumed.

\section{B. Distributed Rework}

In a distributed rework system, some rework stations process all PWA's while other stations only work on PWA's that have failed an in-circuit or functional test. For example, an inspection station located after the component placement machines may have the task of inspecting every PWA to detect components that have been placed inaccurately. The inspector can then push the improperly placed component into the correct position before allowing the PWA to enter the solder station. Farther down the production line another station will rework only those PWA's that have failed the post-solder in-circuit or functional test. The rework effort is very slight for the single component adjusted before soldering and may be quite high for the post-solder failure. However, one must also consider the cost of all the good components inspected in order to account for the total cost involved. Thus the cost model must consider the proportion of PWA's that go through each rework activity.

It was noted earlier that a distributed rework system contains a terminal rework system as its last rework step. Thus the distributed system may be considered to be a terminal system with added, distributed elements. The cost of operating the terminal rework system is shown in the previous section. The cost of operating the distributed rework elements is given by

$$
C_{\mathrm{RW}_{D}}=\sum_{i=1}^{r} t_{\mathrm{RW}_{i}}\left\{W_{\mathrm{RW}_{i}}+\frac{M_{\mathrm{RW}_{i}}+C_{R \cdot \mathrm{Prog}_{i}}}{(7200000 N S)}\right\}
$$

where

$r \quad$ number of added, distributed rework stations,

$M_{\mathrm{RW}_{i}}$ total cost of rework equipment (reflow device, controller, fittings, inspection equipment, part feeders, transfer equipment, etc.) at station $i$,

$W_{\mathrm{RW}_{i}} \quad$ wage paid to rework technician(s) at station $i$,

$t_{\mathrm{RW} i}$ average time to rework a faulty PWA at station $i$,

$C_{R . \text { Prog }_{i}}$ the cost of initial controller programming at station $i$.

The diagnostic step typically only occurs in conjunction with an in-circuit or functional test failure. The distributed rework activities are generally inspection and rework stations which have very few mechanical aids. Typical equipment includes a magnifying glass and a probe or wand and perhaps a simple soldering device. Thus equipment and programming costs are very small. It should be noted that, for the case described, the distributed rework stations only detect faults which are readily observable and easily fixed. Design problems, logic problems, and faulty components can only be detected during the post-solder functional and in-circuit testing.

If we include diagnostic and retest costs, and assume only one retest $\left(x_{C}<0.01\right)$, then the diagnosis and rework cost 
per acceptable PWA is

$$
C D R_{D}=C_{\mathrm{RW}_{D}}+\left(C_{\mathrm{Diag}}+C_{\mathrm{RW}_{T}}+C_{\mathrm{Test}}\right)\left(1-\mathrm{FPY}_{D}\right) \text {. }
$$

\section{Economic Analysis}

Distributed rework is useful if it increases FPY enough to offset the cost of the technicians involved. During a plant trip to a firm which uses the distributed rework strategy, the host remarked that the rework technicians located on the line before the solder process (these inspectors were called "tweakers") represented essentially no cost impact, even though they inspected and reworked every PWA produced. The payoff came in having fewer PWA's fail the post-solder tests, i.e., in having a higher FPY. We may see the economic justification for such a case below.

Based on a component fault rate of $0.3 \%(F R=0.003)$ the 125 components in a hypothetical PWA will produce a FPY of $68.7 \%$. In other words $31.3 \%$ of PWA's produced will have at least one defect. Specifying a payback period of 2 a for capital investments and two shifts working, it is possible to estimate the cost penalty associated with the test, diagnostic, and rework activities.

For a test cycle of $60 \mathrm{~s}, C_{\text {Test }}=\$ 2.44$. This cost is assumed constant for all PWA's of this type, both for postproduction and post-rework tests. For a diagnostic time assumed to be $15 \mathrm{~min}$, and with capital equipment and programming costs and wage rates the same as for the test station, then the cost to diagnose one faulty PWA is $C_{\text {Diag }}=$ $\$ 36.06$.

If we first consider a terminal rework arrangement, then there will be a single rework station located after the test station and after the diagnostic station as shown in Fig. 5. Assuming a capital investment of $\$ 34100$ and a wage rate of $\$ 0.005$ per second ( $\$ 18.00$ per hour) and assuming an average rework time, $t_{\mathrm{RW}}=15 \mathrm{~min}$, then the cost to rework one faulty PWA is $C_{\mathrm{RW}}=\$ 5.89$. However, the important term is $C D R$, the diagnostic and rework cost per acceptable PWA produced. For this case $C D R_{T}=\$ 13.89$.

Now consider a distributed rework environment. For this analysis, the rework arrangement illustrated in Fig. 6 will be modeled. There is a "tweaker" station before the solder station and touchup station after the solder station. Suppose that the PWA must spend $6 \mathrm{~min}$ at each of these stations. Note that this time implies that there may be multiple workers at each of these stations in order to maintain the total production rate. Suppose also that these workers receive the same wage as the terminal rework technician. As mentioned earlier, the distributed stations typically have very few mechanical aids, so assume an equipment cost of $\$ 5000$.

The purpose of the distributed stations in the process is to raise FPY. An interesting question is, "how much improvement in FPY is needed for the distributed workers to pay for themselves?" To answer this question, calculate the FPY required to make $C D R_{D}=C D R_{T}$. Distributed rework costs for this case are $\$ 1.55$ for each station. These costs must be added to each PWA since each PWA passes through the distributed stations. The terminal diagnostic, rework, and retest activities are assumed similar for both systems, so their costs are the same.

Therefore, substituting in (22), one obtains

$$
C D R_{D}=\$ 3.10+(\$ 44.39)\left(1-\mathrm{FPY}_{D}\right) \text {. }
$$

Equating this expression to $C D R_{T}$ yields $\mathrm{FPY}_{D}=0.757$. Thus the distributed rework technicians must raise the FPY from 69 to $76 \%$ to pay for themselves. Further FPY increases represent a cost savings to the firm; hence, the comment that these individuals represent no cost to the firm.

If we look at a case where the initial FPY is higher, say $90 \%$, then the use of distributed rework is harder to justify. In that case the technicians must increase FPY to $97 \%$ before the distributed system saves enough terminal rework expense to pay for the additional workers.

In general, the expression to determine the increase in FPY required to justify distributed rework is derived from (20) and (22). Setting $C D R_{D}=C D R_{T}$ yields

$$
\begin{aligned}
& \left(C_{\text {Diag }}+C_{\mathrm{RW}_{T}}+C_{\text {Test }}\right)\left(1-\mathrm{FPY}_{T}\right)=C_{\mathrm{RW}_{D}} \\
& +\left(C_{\text {Diag }}+C_{\mathrm{RW}_{T}}+C_{\text {Test }}\right)\left(1-\mathrm{FPY}_{D}\right) .
\end{aligned}
$$

Solving for the required change in FPY, $\triangle$ FPY:

$$
\Delta \mathrm{FPY}=\mathrm{FPY}_{D}-\mathrm{FPY}_{T}=\frac{C_{\mathrm{RW}_{D}}}{\left(C_{\text {Diag }}+C_{\mathrm{RW}_{r}}+C_{\text {Test }}\right)} .
$$

In this case, $\triangle \mathrm{FPY}=0.07$. The distributed rework technicians must increase FPY by seven percentage points. It is much easier to go from 69 to $76 \%$ than from 90 to $97 \%$. Hence, we would expect to see a distributed system in cases where the process has low FPY, and a terminal rework system in cases where the process has high FPY.

\section{CONCLUSION}

This paper has presented an analysis of alternate rework strategies for contemporary PWA manufacturing systems involved in batch production. The models used, while based on surface mount technology, may be easily modified to apply to other types of interconnect methods and, indeed, to other industrial processes. We have proposed a method of evaluating whether or not to use distributed, in-process inspection, and touchup personnel in the manufacture of PWA's. It was seen that this decision depends on an evaluation of the effect this strategy has on the FPY of the process. The required change to FPY needed to justify distributed rework was determined. It was seen that for processes with high FPY values, a distributed rework strategy is not warranted, while for processes with low FPY values (e.g., 70\%), the distributed rework effort can be very useful.

\section{REFERENCES}

[1] M. R. Driels and J. S. Klegka, "Analysis of printed wiring board assembly systems," in Proc. Fifth IEEE/CHMT Int. Electronic Manufacturing Technology Symp., 1988, pp. 75-84.

[2] _ 
manufacture using surface mount devices," Int. J. Advanced Manuf. Techn., no. 4, pp. 72-94, 1989.

[3] G. A. Russell, "Design for manufacturability of printed circuit board assemblies," Ann. CIRP, vol. 34, no. 1, pp. 37-40, 1985.

[4] __ "Electronic assembly - The rework issue," in Proc. Second Int. Conf. on Product Design for Manufacture and Assembly, Newport, RI, Apr. 1987

[5] G. Boothroyd, C. Poli, and L. E. Murch, Automatic Assembly. New York: Marcel Dekker, 1982.

[6] G. Boothroyd, "Economics of general-purpose assembly robots," Ann. CIRP, vol. 33, no. 1 , pp. $287-290,1984$

[7] C. Berliner and J. A. Brimson, eds., Cost Management for Today's Advanced Manufacturing. Boston, MA: Harvard Business School, 1988.

[8] S. W. Hinch, Handbook of Surface Mount Technology. Essex, U.K.: Longman Scientific and Technical, 1988.

[9] C. H. Mangin and S. McClelland, Surface Mount Technology, The Future of Electronics Assembly. Bedford, U.K.: IFS, 1987.
[10] R. Truman, "Component assembly onto printed circuit boards," in International Encyclopedia of Robotics, New York: Wiley, 1988.

[11] C. Lea, A Scientific Guide to Surface Mount Technology. Glasgow, Scotland: Electromechanical, 1988

[12] J. A. Crawford, "Milspec repair," in Proc. SMTA Surface Mount 88 Exposition and Conf., 1988, pp. 45-81.

[13] D. Winkler, "Automated electronic module repair," unpublished manuscript, 1988.

[14] J. J. Tuma, Engineering Mathematics Handbook. New York: McGraw Hill, 1987.

[15] U. J. Fan, "Product design analysis for robotic assembly using extended group technology techniques," Ph.D. dissertation, Dep. Mech. Eng., Texas A\&M Univ., 1988.

[16] L. E. Bussey, The Economic Analysis of Industrial Projects. Englewood Cliffs, NJ: Prentice-Hall, 1978.

[17] E. F. Brigham, Financial Management: Theory and Practice. New York: CBS College, 1985. 